\title{
COVID-19 Adaptation of a respiratory service to provide CPAP for patients with COVID-19 pneumonia, outside of a critical care setting, in a district general hospital
}

\author{
Authors: James Talbot-Ponsonby, ${ }^{\mathrm{A}}$ Alvin Shrestha, ${ }^{\mathrm{B}}$ Anitha Vijayasingam, ${ }^{\mathrm{C}}$ Stuart Breck, ${ }^{\mathrm{D}}$ Reza Motazed ${ }^{\mathrm{E}}$ \\ and Yogini Raste ${ }^{F}$
}

\section{Introduction}

One-hundred and forty patients at Croydon University Hospital received continuous positive airway pressure (CPAP) on a specialist respiratory ward, as a bridge to invasive mechanical ventilation (IMV) or as a ceiling of care for COVID-19. This retrospective study aimed to outline service expansion, patient characteristics and explore risk factors in outcomes.

\section{Results}

Mean age of patients on CPAP was 64 years (standard deviation 12). The median number of days from admission to CPAP initiation was 1 day (interquartile range (IQR) $0-3$ ), and time before successful wean off CPAP was 4 days (IQR 2-6). Twenty-eight-day mortality was $64 \%$. Thirty-four per cent of patients went onto require IMV, $24 \%$ improved off CPAP and $41 \%$ were palliated. The $\mathbf{2 8}$-day non-survivor group were of older age, had statistically significant higher admission creatinine and higher peak oxygen requirement. Age above 65 years was associated with higher mortality (odds ratio 5.9; 95\% confidence interval 2.63-13.3).

\section{Conclusion}

CPAP is a viable ceiling-of-treatment option in those unsuitable for ventilation, and may even avoid the need for ventilation in others. Duration on CPAP may be useful for service provision to predict resource allocation. The rapidity from admission to CPAP initiation highlights the need for early ceilings of care to be established.

KEYWORDS: CPAP, SARS-CoV-2, COVID-19, service adaptation, continuous positive airway pressure

DOI: $10.7861 /$ fhj.2020-0270

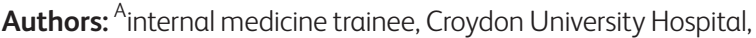
London, UK; ' geriatrics registrar, Croydon University Hospital, London, UK; ${ }^{C}$ respiratory registrar, Croydon University Hospital, London, UK; 'D medical registrar, Croydon University Hospital, London, UK; ${ }^{E}$ Consultant acute physician and nephrologist, Croydon University Hospital, London, UK; ${ }^{\text {F }}$ consultant chest physician, Croydon University Hospital, London, UK

\section{Introduction}

A zoonotic source first gave rise to infections with SARS-CoV-2 causing COVID-19 in humans. This outbreak was declared a global pandemic on 12 March 2020. At the time of writing, there have been over 66 million cases worldwide and more than 1.5 million deaths. ${ }^{2}$ Most cases are asymptomatic or mild, but approximately $5 \%$ are severe of which $26-32 \%$ require ventilatory support in a critical care setting. ${ }^{3}$ Multiple studies have shown non-invasive ventilation (NIV), specifically, continuous positive airway pressure (CPAP) to be efficacious in the treatment of acute respiratory failure (ARF) and in ARF due to a similar coronavirus during the SARS-CoV outbreak in 2003. ${ }^{4-7}$ However, at the start of this pandemic, there were no international guidelines on the use of CPAP in COVID-19. There was initially a reluctance to implement CPAP with a concern regarding aerosolisation of the virus leading to large numbers of infections in healthcare workers. ${ }^{8-10}$ And, as more was learned about the disease, a concern arose regarding COVID-19 subtypes such that CPAP may not be an appropriate treatment for all individuals with COVID-19, risking self-induced lung injury in some. ${ }^{11-13}$ On 06 April 2020, the UK National Institute for Health and Care Excellence published guidance promoting the use of CPAP as a bridge to invasive mechanical ventilation (IMV) only, and advising against the use of high-flow nasal oxygen due to higher risk of infection spread..$^{14}$ The absence of robust evidence or guidelines regarding wider benefits and risks of respiratory interventions for treating this novel disease naturally influenced management, especially in the context of an accelerating epidemic burden.

Croydon University Hospital (CUH) is a busy district general hospital servicing an outer London borough with a large population of nearly 390,000 people. It has a small intensive therapy unit (ITU) of eight beds and seven additional high dependency unit (HDU) beds. An approach of early intubation was initially adopted in attempt to limit disease spread via generation of aerosols associated with CPAP. The rapidity with which individuals requiring critical care presented to $\mathrm{CUH}$ during the first wave of SARS-CoV-2 infections threatened to overwhelm bed capacity. On 01 April 2020, the busiest day of the first wave at CUH, there were 281 inpatients with confirmed COVID-19. This was $132 \%$ more than the average for London NHS hospitals and the sixth highest number of all NHS hospitals in England on this date. ${ }^{15}$ Alongside exponentially increasing cases of severe disease, 
the high failure rate of individuals on IMV was noted and attitudes evolved to include an enthusiasm for trialling CPAP in earnest, both as a bridge to 'buy time' in those that would likely go on to need IMV and as primary treatment for COVID-19. This drive for an alternative to IMV was emboldened by publication of retrospective analyses in China and Italy that had seen high numbers of critically ill patients before the UK. These studies supported the use of CPAP in individuals with ARF, strengthening resolve to meet the rising number of infections with operational changes that definitively involved the use of CPAP. ${ }^{16-18}$ These findings have since been corroborated in other studies and use of CPAP incorporated into international guidelines. ${ }^{19,20}$

We aimed to describe the adaptation of $\mathrm{CUH}$ to manage individuals with COVID-19 with CPAP outside of critical care, to describe the characteristics and any association with mortality.

\section{Methods}

Plans to increase the number of intensive care beds were underway, but difficulties including ensuring appropriate levels of staffing and availability of specialist equipment persisted. The respiratory ward is used to providing NIV for a limited number of individuals depending on staffing. Measures were taken to expand operational capacity of the respiratory ward for safe and effective delivery of CPAP for many critically ill individuals outside of a critical care setting with little time to prepare.

\section{Equipment and staffing}

A large number of CPAP machines (Vivo II and Vivo III) were procured at short notice and tubing adapted to entrain oxygen at higher concentrations. These machines are typically used for delivery of domiciliary CPAP and, as such, have limited capacity for higher fraction of inspired oxygen $\left(\mathrm{FiO}_{2}\right)$. 'Bubble tubing' was used to deliver oxygen at $15 \mathrm{~L} / \mathrm{min}$ through the machine and a second tube delivering the same to the mask, achieving a combined flow of up to $30 \mathrm{~L} / \mathrm{min}$, approximating a maximum $\mathrm{FiO}_{2}$ of $70 \%$.

Individuals requiring CPAP were placed into cohorts of closed bays. Twenty-four-hour senior cover was initiated, including a designated respiratory 'COVID consultant' to discuss referrals for CPAP (available remotely out-of-hours). An additional medical consultant was also available onsite to oversee new admissions and the ongoing management of complex ward issues. The medical junior doctor cover was increased for the respiratory team in-hours and also, overall, for the medical on-call team out-of-hours. The latter allowed, at times, a dedicated 'CPAP doctor' to be present on the respiratory ward to attend to individuals on CPAP immediately. A daily 'COVID update meeting' was introduced attended by hospital management and clinicians on the ground to discuss the ongoing situation and alert stakeholders to arising problems. To guarantee continuing sufficient supply of oxygen to all individuals on CPAP twice daily review of total oxygen use on the two adjacent CPAP wards was undertaken in liaison with the estates department ensuring a maximum flow of $250 \mathrm{~L} / \mathrm{min}$ was not exceeded on either ward at any one time. If there was concern regarding oxygen flow being compromised on one ward individuals were transferred to the ward with a lower oxygen requirement. Regular CPAP machine training and refresher sessions were provided for nurses and doctors expected to manage CPAP.

The critical care outreach team (CCOT) played an important role: ensuring timely initiation of CPAP, transfer of individuals from other wards for treatment with CPAP and attending daily meetings with the on-call respiratory COVID consultant and critical care team to review those deteriorating or failing to improve, and to ultimately assess the requirement for IMV.

\section{Clinical indications for CPAP}

CPAP was considered for individuals with severe type 1 respiratory failure, defined by oxygen saturation $\left(\mathrm{SpO}_{2}\right)$ of $<92 \%$ or respiratory rate $>20$ breaths/min on $60 \%$ inspired oxygen therapy. CPAP treatment was withheld for individuals where contraindicated (eg low consciousness level, low blood pressure or vomiting), in those who needed to be intubated immediately due to clinical severity, in those with significant frailty and/or comorbidity (based on grounds of futility), or due to individual choice. Escalation of individuals on CPAP to IMV was actioned in those deteriorating, defined by worsening oxygenation on arterial blood gas sampling or clinical evidence of tiring with increased work of breathing and/ or unsustainably high respiratory rate.

\section{Data collection and analyses}

Data were then collected retrospectively using electronic health records on individual demographics, and clinical and laboratory parameters. It was, and is still at the time of writing, unknown whether individuals with COVID-19 who were suitable for straightto-IMV should have a trial of CPAP first. Data were also collected to describe mortality in those who went straight-to-IMV and to compare this with CPAP users who went onto IMV. Statistical analyses were performed using Minitab 19 software. Normally distributed data was presented as mean with standard deviation (SD). Non-normally distributed data was presented using median and interquartile range (IQR). Mortality was recorded as 28-day mortality. We had aimed to perform pre-specified analyses of associations between individual characteristics or laboratory parameters and mortality. Chi-squared test was used for categorical data, and binary logistic regression to determine odds ratios. Grouping of variables into categories was decided post hoc. By the time of data collection, all individuals with COVID-19 had reached an endpoint of 'improved', progression to 'IMV' or deemed 'endof-life' (EOL). These endpoints were analysed for association with clinical and laboratory parameters using one-way ANOVA, with post hoc Tukey's test for paired comparison. All statistical tests were two-tailed and statistical significance defined as $p<0.05$.

\section{Results}

Between 07 March 2020 and 03 May 2020, 140 individuals at $\mathrm{CUH}$ received CPAP on the respiratory ward as a bridge to IMV or as a ceiling of care for COVID-19 and, in so doing, helped alleviate bed pressure on ITU. The staff worked to ensure the opportunity of level 2 care for any individual that was failing conservative therapy, it was delivered safely on a standard respiratory ward, limited only by the number of CPAP machines available.

Mean age of individuals on CPAP was 64 years (SD 12); $73 \%$ were men; $27.9 \%$ were White, $25 \%$ were Black, $18.6 \%$ were Asian and $28.5 \%$ of another ethnicity demonstrating an overrepresentation of Black and Asian individuals as compared with the Croydon population. ${ }^{21}$ Ninety per cent were non-frail (by clinical frailty scale $<5) .{ }^{22}$ Eighty-one per cent had a documented treatment escalation plan within 24 hours of admission. The median time from symptom onset to CPAP initiation was 8 days (IQR 6-12) and from admission to CPAP initiation was 1 day (IQR 0-3). Median 
Fig 1. Difference in means between improved, invasive mechanical ventilation and end-of-life outcome groups using one-way ANOVA $(95 \%$ confidence interval for the mean). a) Creatinine vs outcome; $p=0.008$; Tukey's test IMV-EOL. b) Partial pressure of oxygen:fraction of inspired oxygen at start of continuous positive airway pressure vs outcome; $\mathrm{p}=0.006$; Tukey's test IMV-EOL and improved-IMV. c) Highest positive end-expiratory pressure required vs outcome; $p<0.001$; Tukey's test improved-EOL and improved-IMV. d) Highest fraction of inspired oxygen required vs outcome; $p<0.001$; improved-IMV and improved-EOL. CPAP = continuous positive airway pressure; $\mathrm{EOL}=$ end-of-life; $\mathrm{FiO}_{2}=$ fraction of inspired oxygen; IMV = invasive mechanical ventilation; PEEP = positive end-expiratory pressure; P:F = partial pressure
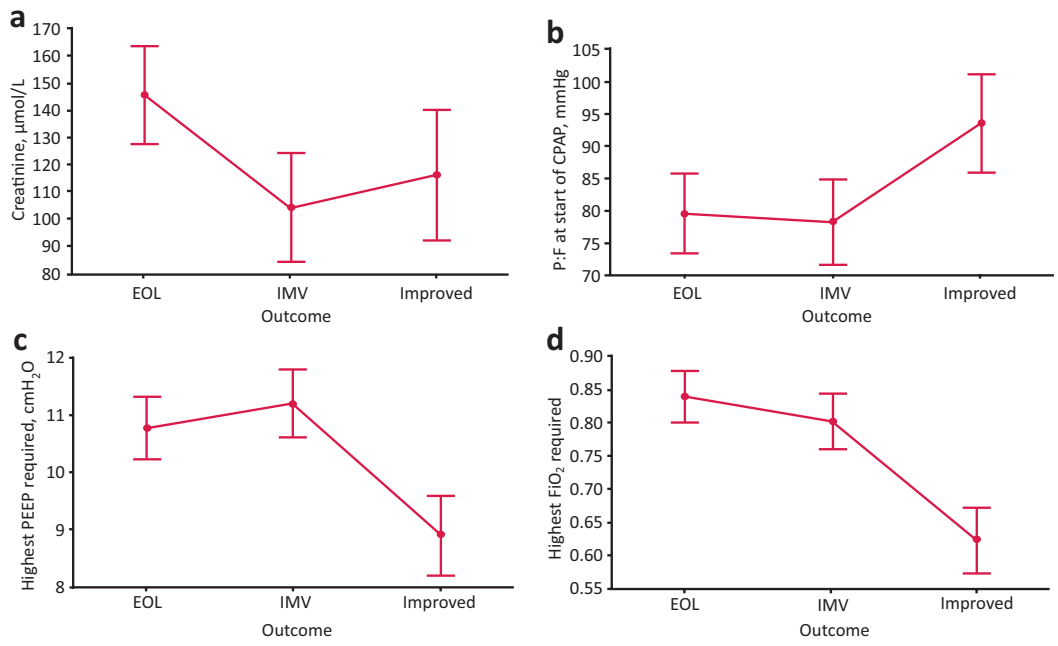
of oxygen:fraction of inspired oxygen.

time before successful wean off CPAP was 4 days (IQR 2-6), while time taken to progress to IMV was 3.25 days (IQR 2-4.25) and to succumb to EOL was 3 days (IQR 2-4.5).

\section{Mortality and outcomes:}

Overall, 28 -day mortality was $63 \%$. Thirty-four per cent $(n=48)$ of individuals treated with CPAP went onto require IMV, $24 \%(n=34)$ improved off CPAP and $41 \%(n=58)$ were deemed EOL and palliated. At 28 days, 33/34 successfully weaned off CPAP were still alive, while all those who were palliated had died. There was a statistically significant difference between groups as determined by one-way ANOVA for admission creatinine $(F(2,134)=4.99$; $p=0.008)$, partial pressure of oxygen:fraction of inspired oxygen $(P: F ; F(2,122)=5.26 ; p=0.006)$, highest positive end-expiratory pressure $(\mathrm{PEEP})$ requirement $(\mathrm{F}(2,137)=13.2 ; \mathrm{p}<0.001)$ and $\mathrm{FiO}_{2}$ requirement $(F(2,135)=24.92 ; p<0.001$; Fig 1$)$.

The 28-day non-survivor group were of older age, had statistically significant higher admission creatinine and higher peak oxygen requirement (Table 1). CPAP intolerance or complication

Table 1. Characteristics of continuous positive airway pressure recipients separated by 28-day mortality

\begin{tabular}{|c|c|c|c|}
\hline & Survived & Died & \\
\hline Individuals with COVID-19, $\mathrm{n}^{\mathrm{a}}$ & 51 & 88 & \\
\hline Swab PCR positive, $n$ & $41 / 51$ & $79 / 85^{b}$ & \\
\hline Mean age, years & 58 & 66 & $\mathrm{p}<0.001$ ( $\mathrm{T}$ test) \\
\hline Male, n (\%) & $37(73)$ & $64(73)$ & \\
\hline Ethnicity, n (\%) & & & $p=0.93$ (chi-squared test) \\
\hline Asian & $9(18)$ & $17(19)$ & \\
\hline Black & $13(25)$ & $21(24)$ & \\
\hline White & $13(25)$ & $26(30)$ & \\
\hline Other & $16(31)$ & $24(27)$ & \\
\hline \multicolumn{4}{|l|}{ Comorbidity, n (\%) } \\
\hline Cardiovascular & $4(8)$ & $16(18)$ & \\
\hline Respiratory & $6(12)$ & $6(7)$ & \\
\hline Hypertension & $25(49)$ & $38(43)$ & \\
\hline Diabetes mellitus & $14(27)$ & $36(41)$ & \\
\hline Clinical frailty scale $<5, \mathrm{n}(\%)$ & $48(94)$ & $77(88)$ & $\mathrm{p}=0.21$ (chi-squared test) \\
\hline Admission creatinine, $\mu \mathrm{mol} / \mathrm{L}$ & 103 & 131 & $p=0.02(T$ test $)$ \\
\hline Admission C-reactive protein, mg/L & 154 & 162 & $p=0.67(T$ test $)$ \\
\hline Highest $\mathrm{FiO}_{2}$ required & 0.69 & 0.83 & $\mathrm{p}<0.001$ ( $T$ test $)$ \\
\hline Highest PEEP required, $\mathrm{cmH}_{2} \mathrm{O}$ & 10.1 & 10.8 & $p=0.13(T$ test $)$ \\
\hline
\end{tabular}

ane data point missing for 28-day mortality, omitted during analysis; 'three data points missing for swab results in 'died' group; CPAP = continuous positive airway pressure; $\mathrm{FiO}_{2}=$ fraction of inspired oxygen; $\mathrm{PCR}=$ polymerase chain reaction; $\mathrm{PEEP}=$ positive end-expiratory pressure. 
Table 2. Comparison of CPAP-to-IMV and straightto-IMV groups

\begin{tabular}{|c|c|c|}
\hline & $\begin{array}{l}\text { CPAP-to-IMV, } \\
\mathrm{n}=48\end{array}$ & $\begin{array}{l}\text { Straight-to- } \\
\mathrm{IMV}, \mathrm{n}=22\end{array}$ \\
\hline Male, n (\%) & $38(79)$ & $14(64)$ \\
\hline Female, n (\%) & $10(20)$ & $8(36)$ \\
\hline Mean age, years & 59 & 54 \\
\hline \multicolumn{3}{|l|}{ Comorbidities, n ( \%) } \\
\hline Hypertension & $19(40)$ & $10(45)$ \\
\hline Diabetes mellitus & $16(33)$ & $10(45)$ \\
\hline Respiratory disease & $4(8)$ & $5(23)$ \\
\hline Cardiac disease & $2(4)$ & 0 \\
\hline 28-day mortality, $n(\%)^{a}$ & $28 / 47(60)$ & $12 / 20(60)$ \\
\hline
\end{tabular}

was associated with EOL (chi-squared test $p=0.01$ ), but not 28-day mortality. Age above 65 years was associated with higher 28-day mortality, conferring an odds ratio of 5.9 (95\% confidence interval (CI) 2.63-13.3) by binary logistic regression.

\section{CPAP-to-IMV vs straight-to-IMV}

There was no difference in 28-day mortality between those requiring IMV after CPAP failure (CPAP-to-IMV) and those who went straight-to-IMV in our institution (chi-square test $p=0.90$; Table 2).

\section{Conclusion}

The extraordinary efforts of the respiratory team and affiliated disciplines of acute medicine, critical care outreach and nursing showed remarkable dynamism in effecting the safe delivery of level 2 care. CPAP appears to be a viable ceiling-of-treatment option in those unsuitable for ventilation and may even avoid the need for ventilation in others; however, mortality remains high. The non-significant difference in 28-day mortality between those requiring IMV after CPAP failure and those who went straight-toIMV may suggest a role for a trial of CPAP before IMV. Although it does compare two groups who are both eligible for IMV, the comparison must be taken with caution given the difference in baseline characteristics in the two groups; in addition, the straight-to-IMV group may have been more unwell, therefore requiring immediate ventilation. Nightingale et al demonstrated, in their study of CPAP at Liverpool University Hospital, a 38\% rate of individuals going on to require IMV and a median duration of 4 days on CPAP, which are both similar to our cohort. ${ }^{23}$ Our 'improved' and successful wean off CPAP rate of $24 \%$ was markedly lower than the aforementioned study, which quoted an 'improvement and discharge from hospital' rate of $55.5 \%$. However, it should be noted the mean age of our individuals with COVID-19 at CUH was 64 years, compared with Liverpool University Hospital's 52 years.

Other learning points from our study include that initial rapid deterioration requiring CPAP (within a median of 1 day from admission) highlights the important priority of early escalation planning which, undeterred by the complexity of the situation, was largely upheld with exemplary timeliness. These are commendable reflections of both the practical actions taken to ensure safe and effective treatment in a non-standard setting and the staff's indefatigable striving for excellence in the face of unprecedented operational pressure. This study also notes that duration on CPAP may be useful for service provision to predict resource allocation, while the described risk factors for mortality may help in prognostication. Palliation of $41 \%$ of individuals with COVID-19 is a stark enumeration stressing the importance of early involvement of palliative care services in those deteriorating or failing to improve.

Limitations of this study include being single-centred, and a lack of control group to appreciate true efficacy. As a retrospective study, it was susceptible to documentation error and occasional missing data. Nevertheless, this study holds one of the largest cohort of individuals who received CPAP for COVID-19 outside of an ITU setting in the UK, and the lessons learnt may be valuable in future surges that threaten to overwhelm our ITU capacity.

\section{References}

1 Zhu N, Zhang D, Wang W et al. A novel coronavirus from patients with pneumonia in China, 2019. N Engl J Med 2020;382:727-33.

2 World Health Organization. WHO Coronavirus (COVID-19) Dashboard. WHO. https://covid19.who.int [Accessed 15 December 2020].

3 Centers for Disease Control and Prevention. Interim clinical guidance for management of patients with confirmed coronavirus disease (COVID-19). CDC, 2020. www.cdc.gov/coronavirus/2019ncov/hcp/clinical-guidance-management-patients.html [Accessed 15 December 2020].

4 Williams T, Finn J, Perkins G et al. Prehospital continuous positive airway pressure for acute respiratory failure: a systematic review and meta-analysis. Prehosp Emerg Care 2013;17:261-73.

5 Bakke S, Botker M, Riddervold I et al. Continuous positive airway pressure and noninvasive ventilation in prehospital treatment of patients with acute respiratory failure: a systematic review of controlled studies. Scand J Trauma Resusc Emerg Med 2014;22:69.

6 Faria D, da Silva E, Atallah Álvaro N et al. Noninvasive positive pressure ventilation for acute respiratory failure following upper abdominal surgery. Cochrane Database Syst Rev 2015:CD009134.

7 Cheung T, Yam L, So L et al. Effectiveness of Noninvasive Positive Pressure Ventilation in the Treatment of Acute Respiratory Failure in Severe Acute Respiratory Syndrome. Chest 2004;126:845-50.

8 Hui D, Hall S, Chan M et al. Noninvasive Positive-Pressure Ventilation: An Experimental Model to Assess Air and Particle Dispersion. Chest 2006;130:730-40.

9 Hui D, Chow B, $\mathrm{Ng} \mathrm{S}$ et al. Exhaled air dispersion distances during noninvasive ventilation via different respironics face masks. Chest 2009;136:998-1005.

10 Arulkumaran N, Brealey D, Howell D et al. Use of non-invasive ventilation for patients with COVID-19: a cause for concern? Lancet Respir Med 2020;8:e45.

11 Marini J, Gattinoni L. Management of COVID-19 Respiratory Distress. JAMA 2020;323:2329-30.

12 Gattinoni L, Coppola S, Cressoni M et al. COVID-19 does not lead to a "typical" acute respiratory distress syndrome. Am J Respir Crit Care Med 2020;201:1299-300.

13 Pan C, Chen L, Lu C et al. Lung recruitability in COVID-19-associated acute respiratory distress syndrome: a single-center observational study. Am J Respir Crit Care Med 2020;201:1294-7.

14 NHS England, NHS Improvement. Guidance for the role and use of non-invasive respiratory support in adult patients with coronavirus (confirmed or suspected). NHS, 2020. www.nice.org.uk/Media/ 
Default/About/COVID-19/Specialty-guides/specialty-guide-NIVrespiratory-support-and-coronavirus.pdf [Accessed 15 December 2020].

15 NHS. COVID-19 Hospital Activity. NHS. www.england.nhs.uk/ statistics/statistical-work-areas/covid-19-hospital-activity [Accessed 15 December2020].

16 Wu Z, McGoogan J. Characteristics of and important lessons from the coronavirus disease 2019 (COVID-19) outbreak in china: summary of a report of 72314 cases from the Chinese Center for Disease Control and Prevention. JAMA 2020;323:1239-42.

17 Giwa A, Desai A, Duca A. Coronavirus SARS-CoV-2 (COVID-19): an overview for emergency clinicians. EB Medicine, 2019. www. ebmedicine.net/topics/infectious-disease/COVID-19 [Accessed 15 December 2020]

18 Wang K, Zhao W, Li ] et al. The experience of high-flow nasal cannula in hospitalized patients with 2019 novel coronavirus-infected pneumonia in two hospitals of Chongqing, China. Ann Intensive Care 2020;10:37.

19 Ashish A, Unsworth A, Martindale ] et al. CPAP management of COVID-19 respiratory failure: a first quantitative analysis from an inpatient service evaluation. BMJ Open Respir Res 2020;7:e000692.
20 World Health Organization. Clinical management of severe acute respiratory infection when COVID-19 is suspected. WHO, 2020. www.who.int/publications-detail/clinical-management-of-severeacute-respiratory-infection-when-novel-coronavirus-(ncov)-infectionis-suspected [Accessed 15 December 2020].

21 Croydon Observatory. Population. Croydon Observatory. www. croydonobservatory.org/population [Accessed 25 January 2020].

22 Church S, Rogers E, Rockwood $\mathrm{K}$ et al. A scoping review of the clinical frailty scale. BMC Geriatr 2020;20:393.

23 Nightingale R, Nwosu N, Kutubudin F et al. Is continuous positive airway pressure (CPAP) a new standard of care for type 1 respiratory failure in COVID-19 patients? A retrospective observational study of a dedicated COVID-19 CPAP service. BMJ Open Respir Res 2020;7:e000639.

Address for correspondence: Dr James Talbot-Ponsonby, Croydon University Hospital, 530 London Road, Thornton Heath, Croydon, London CR7 7YE, UK.

Email: james.talbot1@nhs.net 